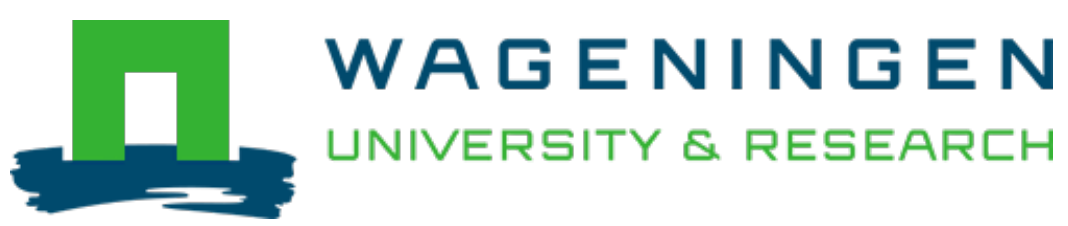

\title{
Positive effects of folic acid supplementation on cognitive aging are dependent on $\omega-3$ fatty acid status : a post hoc analysis of the FACIT trial
}

\author{
American Journal of Clinical Nutrition \\ Soest, Annick P.M.; Rest, Ondine; Witkamp, Renger F.; Groot, Lisette C.P.G.M. \\ https://doi.org/10.1093/ajcn/nqaa373
}

This article is made publicly available in the institutional repository of Wageningen University and Research, under the terms of article $25 \mathrm{fa}$ of the Dutch Copyright Act, also known as the Amendment Taverne. This has been done with explicit consent by the author.

Article 25 fa states that the author of a short scientific work funded either wholly or partially by Dutch public funds is entitled to make that work publicly available for no consideration following a reasonable period of time after the work was first published, provided that clear reference is made to the source of the first publication of the work.

This publication is distributed under The Association of Universities in the Netherlands (VSNU) 'Article $25 \mathrm{fa}$ implementation' project. In this project research outputs of researchers employed by Dutch Universities that comply with the legal requirements of Article $25 \mathrm{fa}$ of the Dutch Copyright Act are distributed online and free of cost or other barriers in institutional repositories. Research outputs are distributed six months after their first online publication in the original published version and with proper attribution to the source of the original publication.

You are permitted to download and use the publication for personal purposes. All rights remain with the author(s) and / or copyright owner(s) of this work. Any use of the publication or parts of it other than authorised under article $25 \mathrm{fa}$ of the Dutch Copyright act is prohibited. Wageningen University \& Research and the author(s) of this publication shall not be held responsible or liable for any damages resulting from your (re)use of this publication.

For questions regarding the public availability of this article please contact openscience.library@wur.nl 


\title{
Positive effects of folic acid supplementation on cognitive aging are dependent on $\omega-3$ fatty acid status: a post hoc analysis of the FACIT trial
}

\author{
Annick PM van Soest, Ondine van de Rest, Renger F Witkamp, and Lisette CPGM de Groot
}

Division of Human Nutrition and Health, Wageningen University and Research, Wageningen, The Netherlands

\begin{abstract}
Background: Although epidemiological studies suggest a protective role of B vitamins and omega-3 ( $\omega-3)$ fatty acids in cognitive decline, findings from intervention studies are conflicting. Mechanistic studies suggest that the $\omega-3$ (n-3) fatty acid status can modulate the effects of $\mathrm{B}$ vitamins on cognitive decline.

Objectives: We investigated the interaction between baseline $\omega-3$ fatty acid statuses and folic acid treatment on cognitive decline in a placebo-controlled trial [FACIT (Folic Acid and Carotid Intimamedia Thickness)].
\end{abstract}

Methods: This post hoc analysis included 791 older adults aged 50-70 y with plasma total homocysteine $\geq 13 \mu \mathrm{mol} / \mathrm{L}$ and $\leq 26 \mu \mathrm{mol} / \mathrm{L}$ and serum vitamin B12 $\geq 200 \mathrm{pmol} / \mathrm{L}$. Participants received $800 \mu \mathrm{g}$ folic acid or placebo daily for $3 \mathrm{y}$. Global cognitive functioning and domain-specific functioning (episodic memory, information processing speed, executive functioning) were assessed at baseline and after $3 \mathrm{y}$. The effect of the folic acid supplementation was analyzed according to tertiles of baseline $\omega-3$ fatty acid concentrations using linear multiple regression.

Results: The mean \pm SD age of the study population was $60.2 \pm$ $5.6 \mathrm{y}$, and the mean \pm SD Mini-Mental State Examination score was $28.6 \pm 1.5$. The treatment effect of folic acid was significantly larger in participants in the low compared to high $\omega-3$ fatty acid tertile for global cognition (difference in z-score: mean $\pm \mathrm{SE}=0.16 \pm 0.059$; $P<0.01$ ). Regarding domain-specific functioning, similar results were observed for information processing speed (mean $\pm \mathrm{SE}=0.167$ $\pm 0.068 ; P=0.01$ ). There were no overall interactions between folic acid treatment and $\omega-3$ fatty acid tertiles for episodic memory $(P=0.14)$ and executive functioning $(P=0.21)$.

Conclusions: This post hoc analysis revealed that the efficacy of folic acid treatment on cognitive functioning is dependent on the $\omega-3$ fatty acid status. Individuals with a lower $\omega-3$ fatty acid status at baseline benefited from folic acid treatment, while individuals with a higher $\omega$-3 fatty acid status did not. The results potentially explain the inconsistency in outcomes of B-vitamin supplementation trials and emphasize the importance of a personalized approach. This trial was registered at clinicaltrials.gov as NCT00110604. Am J Clin Nutr 2021;113:801-809.

Keywords: B vitamins, cognitive functioning, folic acid, omega-3 fatty acids, older adults, elderly, healthy aging

\section{Introduction}

Dementia and cognitive decline are a public health priority. Due to population aging, the number of people suffering from dementia is expected to triple in the upcoming $30 \mathrm{y}$, to 150 million in 2050. This steep increase in prevalence will pose a great social and economic impact on caregivers, families, and society (1). The great burden and impact, along with the lack of effective treatment options, create an urgent need for strategies to prevent or slow down disease progression at an early stage. Nutritional interventions are considered promising strategies, with special interest for B vitamins and $\omega-3$ fatty acids (2).

Inadequate concentrations of the B vitamins folic acid, B6, and $\mathrm{B} 12$ result in accumulation of the amino acid homocysteine,

Supported by the Wageningen Centre for Food Sciences, Wageningen University, and The Netherlands Organization for Health Research and Development (ZonMW, grant number 20010002).

Supplemental Figure 1 and Tables 1 and 2 are available from the "Supplementary data" link in the online posting of the article and from the same link in the online table of contents at https://academic.oup.com/ajcn/.

Address correspondence to APMvS (e-mail: annick.vansoest@wur.nl).

Abbreviations used: AD, Alzheimer's disease; CST, concept shifting test; FACIT, Folic Acid and Carotid Intima-media Thickness; MCI, mild cognitive impairment; PC, phosphatidylcholine; SAH, S-adenosyl homocysteine; VITACOG, Homocysteine and B-vitamins in Cognitive Impairment.

Received June 3, 2020. Accepted for publication November 16, 2020.

First published online February 10, 2021; doi: https://doi.org/10.1093/ajcn/ nqaa373. 
which is a risk factor for cognitive decline and dementia (3, 4). Supplementation with these B vitamins has been shown to lower homocysteine concentrations, yet studies regarding the effectiveness of B-vitamin supplementation on cognitive decline show conflicting results (5). Similarly, there is no consensus on the role of $\omega-3$ fatty acids in cognitive decline and dementia. Although epidemiological studies support their protective role in cognitive decline $(6,7)$, intervention studies on $\omega$-3 fatty acid supplementation are inconsistent (8).

The extent of cognitive impairments and baseline nutrient and homocysteine statuses are important factors explaining these mixed results (9-11), but more factors are thought to be involved. Interestingly, mechanistic studies suggest a link between $\omega$-3 fatty acids and homocysteine at the level of phospholipid metabolism (12). Therefore, it is hypothesized that sufficient availability of both $\omega-3$ fatty acids and B vitamins is crucial to inhibit the neuropathology underlining age-related cognitive decline and dementia.

Preliminary proof supporting this hypothesis comes from 2 studies showing that supplementation with a combination of vitamin B6, vitamin B12, and folic acid was more effective in slowing down cognitive decline (13) and brain atrophy (14) in subjects with mild cognitive impairment (MCI) who had a high compared to a low $\omega-3$ fatty acid status. In line with this, Jernerén and colleagues (15) showed that positive effects of $\omega-3$ fatty acid supplementation on cognitive functioning in Alzheimer's disease $(\mathrm{AD})$ subjects were only present in individuals who had lower homocysteine concentrations at baseline. The current analysis was undertaken to further investigate this interaction and to study whether it also occurs in healthy older adults without cognitive complaints. To this end, we investigated the interaction between baseline $\omega$ - 3 fatty acid statuses and folic acid treatment on cognitive decline in healthy older adults with elevated homocysteine concentrations in the FACIT trial (Folic Acid and Carotid Intima-media Thickness).

\section{Methods}

\section{Study design and participants}

Data used for this post hoc analysis are from the FACIT study, a randomized, double-blind, placebo-controlled trial investigating the effect of folic acid supplementation on carotid-intima-media thickness. Cognitive functioning was measured as a secondary outcome. Data were collected between 2000 and 2004 in the Netherlands. The trial has been registered at clinicaltrials.gov as NCT00110604. The study has been approved by the Medical Ethics committee from Wageningen University and Research, and participants have given written informed consent. Details on recruitment and participants have been described previously (16). Briefly, 819 men and postmenopausal women aged 50-70 y with elevated plasma total homocysteine concentrations $(\geq 13 \mu \mathrm{mol} / \mathrm{L})$ were randomly assigned to either supplementation with $800 \mu \mathrm{g}$ folic acid or placebo once daily for $3 \mathrm{y}$. Participants with plasma homocysteine concentrations $>26 \mu \mathrm{mol} / \mathrm{L}$ or serum vitamin B12 concentrations $<200 \mathrm{pmol}$ were excluded. Our analysis included data of 791 participants. Data from 28 participants were excluded due to missing fatty acid $(n=11)$, cognitive testing $(n=1)$, or follow-up ( $n=16)$ data (Supplemental Figure 1).

\section{Cognitive testing}

Cognitive performance was assessed at baseline and after $3 \mathrm{y}$ of intervention with a battery of 5 cognitive tests derived from the Maastricht Ageing Study (17).

\section{Concept shifting test.}

The concept shifting test (CST) (18) assesses the ease of concept switching and is a measure of executive functioning. The test comprised 4 subtests in which the participant was presented with a screen containing 16 small circles arranged in a large circle. In the 4 consecutive subtests, the small circles were either empty or contained a letter, a number, or both letters and numbers. Participants were instructed to cross out the numbers in chronological order (part A), to cross out the letters in alphabetical order (part B), to alternate crossing out numbers and letters in chronological and alphabetical order (part C), and to cross out all empty circles in any random order (subtest blank). The time to complete each of the 4 subtasks was documented.

\section{Stroop Color-Word test.}

The Stroop Color-Word test (19) assesses cognitive interference and is a measure of executive functioning. The test consisted of 3 subtests, in which the participant was presented with stimuli of either written color names or colored blocks. The participant was asked to name the color names printed in black ink (subtest words), the color of the blocks (subtest colors), and the color of the ink while this color was incongruent with the written word (subtest colored words). The time to complete each of the 3 subsets was measured.

\section{Word learning test.}

The word learning test (20) is a measure of memory. A total of 15 monosyllabic words were shown in a fixed sequence for $2 \mathrm{~s}$ each. Immediately after presentation, the participants were asked to recall the words. This procedure was repeated 3 times (immediate recall). At $20 \mathrm{~min}$ after the last presentation of the words, the participant was asked to recall the words again (delayed recall). The number of correctly recalled words was recorded.

\section{Letter digit substitution test.}

The letter digit substitution test (21) assesses information processing speed. In this test, 9 different letters are paired with the numbers 1 to 9 . The participants were shown a random series of letters and were asked to match the corresponding number to the letter as quickly as possible. The number of correctly matched numbers and letters in $90 \mathrm{~s}$ was documented.

\section{Verbal Fluency Test.}

The Verbal Fluency Test (22) measures verbal functioning. The participants were given 1 min to name as many animals as possible. The number of uniquely named animals was documented. 


\section{Biochemical assays}

At baseline, blood was obtained via venipuncture after an overnight fast and collected in Vacutainer EDTA tubes. Following centrifuging, plasma was obtained and stored within $2 \mathrm{~h}$ at $-80^{\circ} \mathrm{C}$ until analysis. Concentrations of fatty acids were measured in plasma cholesteryl esters by GC using a modified version of a previously described protocol (6). In short, $650 \mu \mathrm{L}$ EDTA plasma was extracted using hexane, followed by isolation of the cholesteryl fraction by solid phase extraction using silica columns. Subsequently, fatty acids in cholesteryl esters were trans-methylated using sulphuric acid in methanol. After extraction with hexane, individual fatty acid methyl-esters were separated by GC and detected by flame ionization. Peaks were identified based on comparison of retention times to known standards. Data on $\omega-3$ fatty acids as methyl esters are presented in relative concentrations of total fatty acid methyl esters. Total relative plasma $\omega-3$ fatty acids were derived by adding the relative concentrations of EPA and DHA. The inter-assay coefficients of variability for EPA and DHA were $4.35 \%$ and $4.62 \%$, respectively.

Apolipoprotein genotype was determined by PCR of DNA with the restriction enzyme HhaI (23). Plasma total homocysteine and vitamin B6 were measured using $\operatorname{HPLC}(24,25)$. Chemiluminescent immunoassays were used for the determination of vitamin B12 and folate concentrations (Immulite 2000; Diagnostic Products Corporation).

\section{Statistical analysis}

Cognitive composite scores were created to limit the number of dependent outcomes and reduce the chance of a type I error. $\mathrm{Z}$-scores for each cognitive test at baseline and after $3 \mathrm{y}$ of followup were standardized to the mean and SD at baseline. Z-scores for the CST and Stroop Color-Word test were reversed, as lower outcomes for these tests represent better cognitive functioning. The individual tests' $\mathrm{z}$-scores were clustered into global cognition and 3 domain-specific z-scores:

$$
\begin{aligned}
& \text { Global cognition }=\left(\left(z W L T_{\text {total }}+z W L T_{\text {max }}+z W L T_{\text {delayed }}\right.\right. \\
& +-z C S T_{\text {numbers\&letters }}+-z \text { Stroop } p_{\text {words\&colors }}+z L D S T \\
& \left.+-z \text { Stroop } \text { interference }+-z C S T_{\text {shift }}+z \text { Verbal Fluency }\right) / 9
\end{aligned}
$$

Episodic memory $=\left(z W L T_{\text {immediate total }}+z W L T_{\text {immediate max }}\right.$

$$
\left.+z W L T_{\text {delayed }}\right) / 3
$$

Information processing speed $=$

$$
\left(-z C S T_{\text {numbers\&letters }}+-z \text { Stroop }{ }_{\text {words } \& \text { colors }}+z L D S T\right) / 3(3)
$$

Executive functioning $=\left(-z\right.$ Stroop $_{\text {interference }}+-z C S T_{\text {shift }}$

$$
+ \text { zVerbal Fluency)/3 }
$$

Stroop interference, Stroop words and colors, CST shift, and CST numbers and letters were calculated as follows:

$$
\begin{aligned}
\text { Stroop }_{\text {interference }}= & \text { Stroop }_{\text {colored words }} \\
& -\frac{\text { Stroop }_{\text {words }}+\text { Stroop }_{\text {colors }}}{2}
\end{aligned}
$$

$$
\begin{gathered}
\text { Stroop }_{\text {words\&colors }}=\frac{\text { Stroop }_{\text {words }}+\text { Stroop }_{\text {colors }}}{2} \\
C S T_{\text {shift }}=C S T_{\text {partC }}-C S T_{\text {blank }} \\
-\frac{C S T_{\text {part A }}-C S T_{\text {blank }}+C S T_{\text {part B }}-C S T_{\text {blank }}}{2} \\
C S T_{\text {numbers\&letters }}=\frac{C S T_{\text {part A }}-C S T_{\text {blank }}+C S T_{\text {part B }}-C S T_{\text {blank }}}{2}
\end{gathered}
$$

Data are expressed as either $n(\%)$ or mean \pm SD, unless otherwise stated. Differences in baseline characteristics between groups were analyzed using independent sample $t$-tests and an ANOVA for continuous variables and chi-square tests for categorical variables. Multiple linear regression was used to investigate whether the $\omega-3$ fatty acid status modifies the treatment effect of folic acid. The change in z-score between baseline and after 3 y of follow-up for each specific domain was modelled as a function of treatment (folic acid or placebo), baseline $\omega-3$ fatty acid status (low, middle, high), and their interaction. The $\omega-3$ fatty acid status was based on tertiles of baseline $\omega-3$ fatty acid concentrations. To investigate whether the interaction was driven by either EPA or DHA, additional analyses were run using baseline EPA and DHA statuses separately instead of the baseline $\omega-3$ fatty acid status. Moreover, additional analyses including apoE4 status in the interaction were run to determine the role of the apoE4 carrier status in explaining the interaction. The final model was adjusted for the covariates of baseline cognitive $\mathrm{Z}$-score, age, sex, level of education (divided into 3 groups), apoE4 status, baseline homocysteine concentration, and baseline BMI. The covariates of physical activity, cardiovascular disease, and diabetes mellitus were assessed but are not included in the model, as they did not lead to improvements. Tukey correction was used for multiple testing of the treatment effects within the $\omega-3$ fatty acid tertiles. We removed 3 strongly deviating individual test $\mathrm{z}$-scores with values of $\leq-10$ from the analysis to warrant normality of residuals and heterogeneity of variance. Running the analysis including these values did not alter conclusions. A $P$ value of $<0.05$ was considered significant, with the exception of $P$ values for interactions, where $P<0.1$ was considered significant. All statistical analyses were performed using RStudio Version 1.1.463 (26).

\section{Results}

\section{Participant characteristics}

Baseline characteristics of the 791 participants are presented in Table 1. The mean age of the total study population was $60.2 \pm 5.6 \mathrm{y}$, and $71.4 \%$ of the participants were male. The mean baseline homocysteine concentration was $13.3 \pm 2.9 \mu \mathrm{mol} / \mathrm{L}$ and the mean Mini-Mental State Examination score was $28.6 \pm 1.5$. A larger proportion of the participants in the folic acid group had received a low level of education $(P=0.021)$ and suffered from cardiovascular disease $(P=0.035)$ compared to participants in 
TABLE 1 Baseline characteristics per treatment group in the FACIT study ${ }^{1}$

\begin{tabular}{|c|c|c|c|c|}
\hline Characteristic & Overall, $n=791$ & Folic acid, $n=391$ & Placebo, $n=400$ & $P$ value \\
\hline \multicolumn{5}{|l|}{ Sex, $n(\%)$} \\
\hline Female & $226(28.6 \%)$ & $109(27.9 \%)$ & $117(29.3 \%)$ & 0.73 \\
\hline \multicolumn{5}{|l|}{ Level of education, $n(\%)$} \\
\hline Low & $178(22.5 \%)$ & $104(26.6 \%)$ & $74(18.5 \%)$ & \multirow[t]{2}{*}{0.02} \\
\hline Middle & $300(37.9 \%)$ & $137(35.0 \%)$ & $163(40.8 \%)$ & \\
\hline Physical activity, PASE score & $153 \pm 69$ & $154 \pm 71$ & $153 \pm 68$ & 0.82 \\
\hline Current smoker, $n(\%)$ & $159(20.1 \%)$ & $81(20.7 \%)$ & $78(19.5 \%)$ & 0.74 \\
\hline Diabetes mellitus, $n(\%)$ & $24(3.0 \%)$ & $10(2.6 \%)$ & $14(3.5 \%)$ & 0.57 \\
\hline Cardiovascular disease, $n(\%)$ & $93(11.8 \%)$ & $56(14.3 \%)$ & $37(9.3 \%)$ & 0.04 \\
\hline ApoE4 carriers, $n(\%)$ & $248(30.9 \%)$ & $124(31.8 \%)$ & $124(31.2 \%)$ & 0.93 \\
\hline Total homocysteine, $\mu \mathrm{mol} / \mathrm{L}$ & $13.3 \pm 2.9$ & $13.3 \pm 2.6$ & $13.3 \pm 3.1$ & 0.99 \\
\hline $\mathrm{EPA}, 2 \%$ & $1.11 \pm 0.69$ & $1.14 \pm 0.76$ & $1.08 \pm 0.61$ & 0.26 \\
\hline Sum EPA and DHA, ${ }^{2} \%$ & $2.32 \pm 0.86$ & $2.34 \pm 0.95$ & $2.30 \pm 0.77$ & 0.46 \\
\hline MMSE & $28.6 \pm 1.5$ & $28.6 \pm 1.3$ & $28.5 \pm 1.7$ & 0.32 \\
\hline Global cognition z-score & $0.00 \pm 0.66$ & $0.02 \pm 0.67$ & $-0.04 \pm 0.66$ & 0.24 \\
\hline Episodic memory z-score & $0.00 \pm 0.94$ & $0.02 \pm 0.67$ & $-0.04 \pm 0.66$ & 0.24 \\
\hline Information processing speed z-score & $0.00 \pm 0.84$ & $0.03 \pm 0.80$ & $-0.03 \pm 0.88$ & 0.30 \\
\hline Executive functioning $\mathrm{z}$-score & $0.00 \pm 0.84$ & $0.04 \pm 0.73$ & $-0.04 \pm 0.73$ & 0.16 \\
\hline
\end{tabular}

${ }^{1}$ FACIT subjects with available fatty acid and cognition data at both time points. Data are shown as either mean \pm SD or $n$ (\%). FACIT, Folic Acid and Carotid Intima-media Thickness; MMSE, Mini-Mental State Examination; PASE, Physical Activity Scale for the Elderly.

${ }^{2}$ Measured in cholesteryl esters.

the placebo group. There were no further differences in baseline characteristics between intervention groups. With respect to $\omega-3$ fatty acid status, participants in the middle $\omega$-3 fatty acid tertile were more physically active $(P<0.01)$, while higher baseline vitamin B12 concentrations were observed in participants in the high $\omega-3$ fatty acid tertile $(P=0.012$; Supplemental Table 1). Mean baseline cognitive scores for global cognition and all 3 cognitive domains (episodic memory, information processing speed, and executive functioning) did not differ between either intervention or baseline $\omega-3$ fatty acid status groups.

\section{Cognitive performance}

\section{Global cognition.}

Global cognition improved over the 3-y study period in all groups. Folic acid treatment was more effective than placebo in improving global cognition in subjects in the low $\omega$-3 fatty acid tertile (diff mean $\pm \mathrm{SEM}=0.155 \pm 0.042 ; P<0.01$; Table 2). No difference in improvement in global cognition was observed between folic acid and placebo treatment in the middle and high $\omega$-3 fatty acid tertiles [diff $=0.096 \pm 0.042(P=0.19)$ and $-0.008 \pm 0.041(P=1.00)$, respectively]. Furthermore, the interaction between folic acid treatment and $\omega$-3 fatty acid tertile was significant $(P=0.02)$. Compared to subjects in the high baseline $\omega-3$ fatty acid tertile, the folic acid treatment effect was significantly larger in subjects within the low $\omega-3$ fatty acid tertile (diff $=0.163 \pm 0.059 ; P<0.01$; Table 2; Figure 1A).
When focusing on EPA and DHA separately, we only observed a significant interaction between folic acid treatment and EPA tertile $(P<0.01$; Supplemental Table 2). The folic acid treatment was significantly larger in subjects in the low and middle EPA tertiles compared to subjects in the high EPA tertile [diff $=0.182 \pm 0.059(P<0.01)$ and diff $=0.118 \pm 0.058$ $(P=0.04)$, respectively]. There was no significant overall interaction between folic acid treatment and DHA tertile $(P=0.104)$.

\section{Domain-specific cognition.}

Episodic memory. Despite the apparently larger differences in treatment effects in subjects in the low and middle $\omega-3$ fatty acid tertiles compared to subjects in the high tertile [diff $=0.216 \pm 0.120(P=0.07)$ and $0.190 \pm 0.120(P=0.11)$, respectively; Table 2; Figure 1B], there was no significant overall interaction between folic acid treatment and $\omega$-3 fatty acid tertile $(P=0.14)$.

Information processing speed. Information processing speed declined during the observation period in all groups, as indicated by the negative changes in z-scores. The folic acid treatment was more effective than placebo in slowing the decline in information processing speed in subjects in the low $\omega$-3 fatty acid tertile (diff $=0.148 \pm 0.049 ; P=0.03$ ), while no difference in treatment effectiveness was observed in subjects in the middle or high $\omega$ 3 fatty acid tertiles ( $P=0.54$ and 1.00 , respectively; Table 2 ). The overall interaction between folic acid treatment and $\omega$-3 fatty 


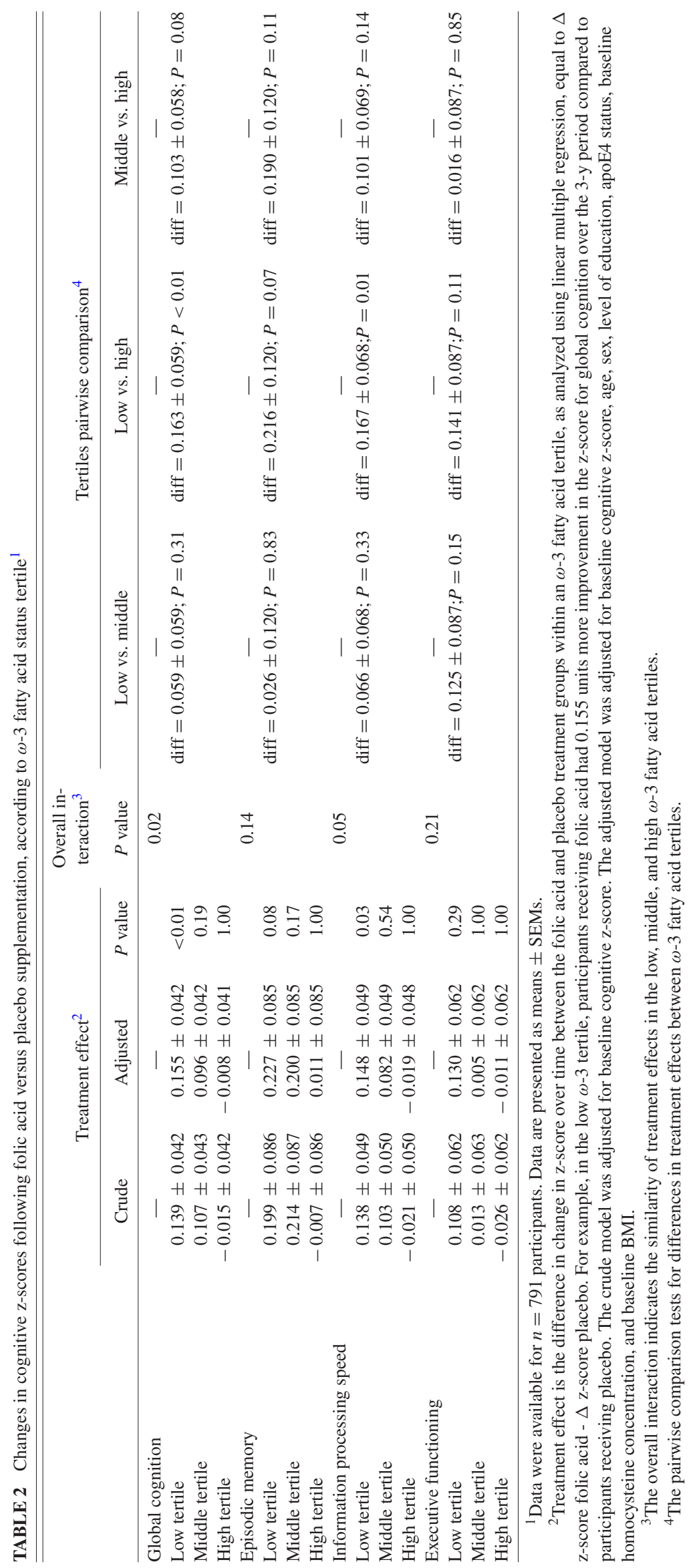


A

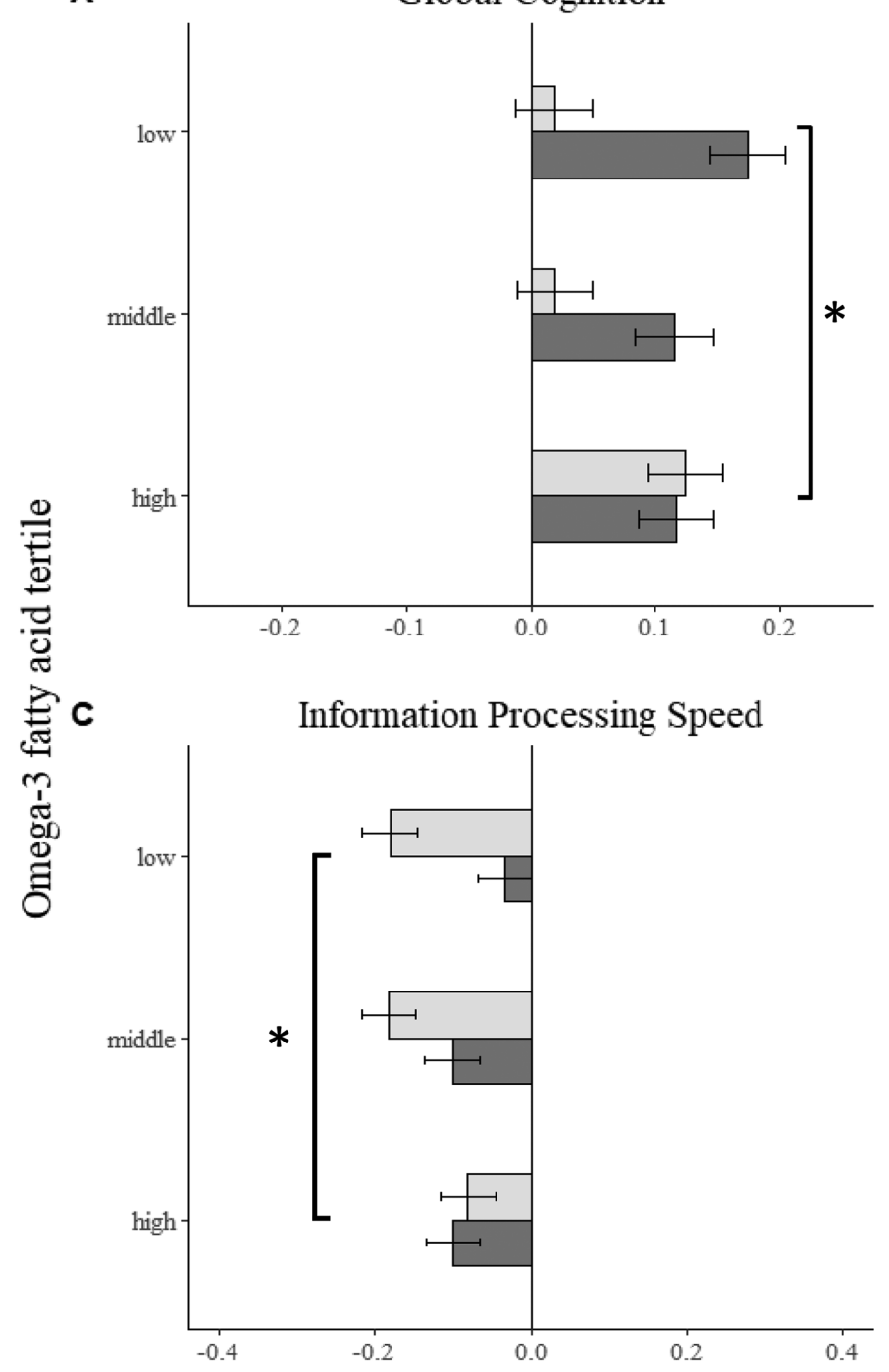

B

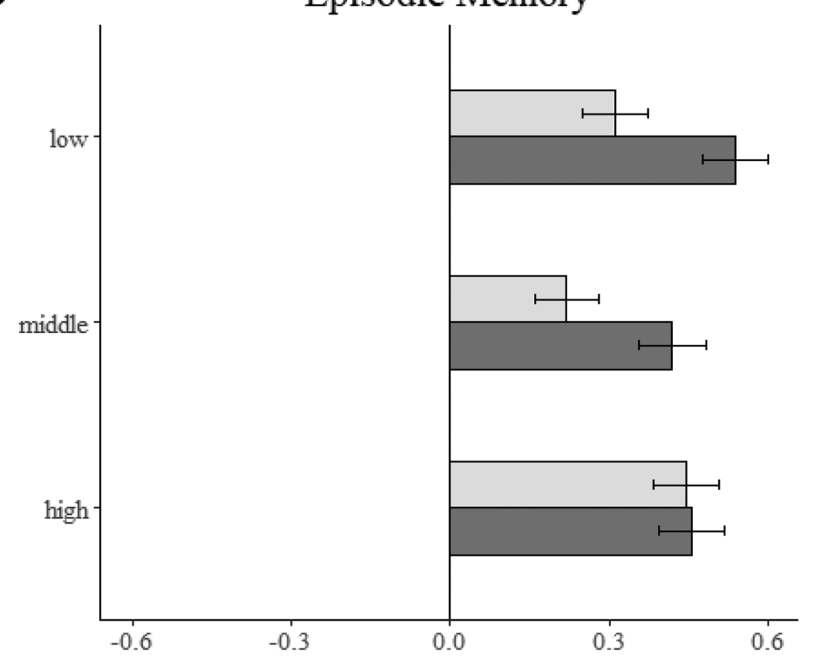

D

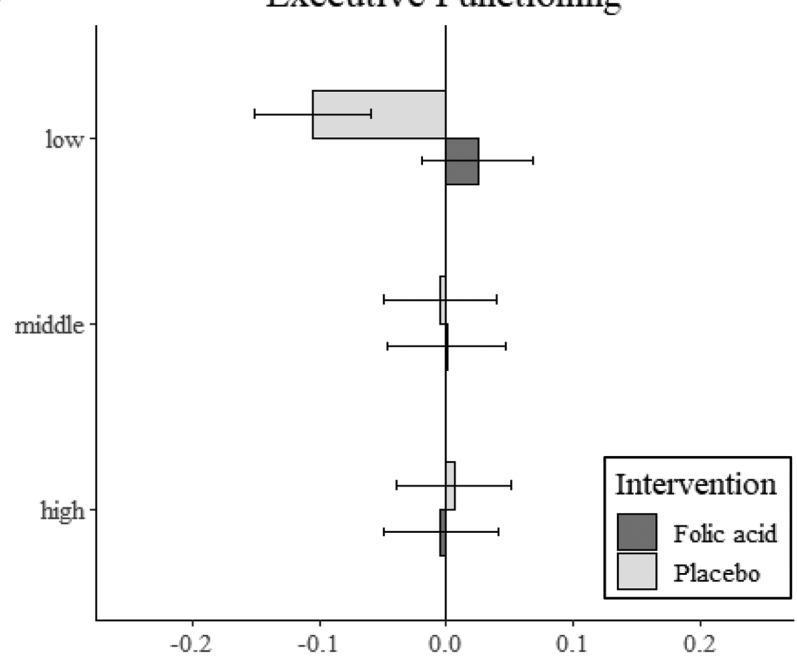

\section{Change in z-score}

FIGURE 1 Changes in z-scores (mean \pm SE) over the 3-y intervention period for (A) global cognition and (B-D) the 3 cognitive domains according to intervention group and $\omega-3$ fatty acid status $(n=791)$. ${ }^{*}$ Significant $(P<0.05)$ difference in treatment effect between $\omega-3$ fatty acid tertiles, as analyzed by linear multiple regression.

acid status was significant $(P=0.05)$. This was due to a larger treatment effect in the low compared to high $\omega$-3 fatty acid tertile (diff $=0.167 \pm 0.068 ; P=0.01$; Table 2; Figure 1C).

Executive functioning. Comparing folic acid and placebo treatment, no treatment effect was observed in any of the $\omega-3$ fatty acid groups. Furthermore, there was no significant overall interaction between folic acid treatment and $\omega$-3 fatty acid status $(P=0.21$; Table 2; Figure 1D).

ApoE4 status. The interaction between folic acid treatment and $\omega$-3 fatty acid tertile on global cognition was not explained by apoE4 status $(P=0.14$; data not shown). Similarly, apoE4 status did not affect the interaction with episodic memory $(P=0.17)$, information processing speed $(P=0.58)$, or executive functioning $(P=0.88)$.

\section{Discussion}

This post hoc analysis revealed that the efficacy of folic acid treatment on cognitive functioning in healthy older adults is dependent on $\omega$-3 fatty acid status. With respect to global cognition, individuals in the low $\omega-3$ fatty acid tertile benefited from the folic acid treatment, while individuals in the high $\omega-3$ fatty acid tertile did not experience an advantage. The interaction is mainly driven by EPA. Regarding domain-specific performance, the effectiveness of folic acid treatment was dependent on baseline $\omega-3$ fatty acid concentrations for information processing speed, but not for episodic memory and executive functioning.

In contrast to our study, previous post hoc analyses on the interactions between $\mathrm{B}$ vitamins and $\omega-3$ fatty acids were performed in persons with impaired cognitive functioning (13-15). Remarkably, opposite effects were observed in the VITACOG 
(Homocysteine and B-vitamins in Cognitive Impairment) trial $(13,14)$, a randomized controlled trial on the effect of $2 y$ of daily supplementation with folic acid, vitamin B6, and vitamin B12 compared with placebo in older adults $(>70 \mathrm{y})$ with MCI. This study showed that B-vitamin supplementation was beneficial in slowing down cognitive decline (13) and brain atrophy rates (14) in individuals with a higher $\omega-3$ fatty acid status, while individuals with a lower $\omega-3$ fatty acid status did not benefit from extra B vitamins. Similarly, a post hoc analysis from the OmegAD trial, in which AD patients were supplemented with EPA and DHA compared with placebo for 6 mo, showed that baseline homocysteine concentrations influence the effectiveness of $\omega-3$ fatty acid supplementation on cognitive functioning, with only individuals with lower homocysteine concentrations benefiting from $\omega-3$ fatty acid supplementation (15).

The difference in study populations may explain the opposite findings. While our study included cognitively healthy older adults aged 50-70 y, the VITACOG and OmegAD trials focused on older participants $(>70$ y) with $\mathrm{MCI}$ and AD. Older cognitively impaired persons may have higher needs for $\omega-3$ fatty acids, due to changes in dietary intake and bioavailability (27). Additionally, the membrane synthesis rate may be higher in response to increased neuronal tissue loss (27-29). Hence, a higher neuronal turnover rate in MCI and $\mathrm{AD}$ patients may increase the need for $\omega$-3 fatty acids. Phosphatidylcholine (PC) is 1 of the carriers involved in the transport of $\omega-3$ fatty acids to the brain. It has been argued that PC concentrations can become a limiting factor resulting in insufficient transport of $\omega-3$ fatty acids to the brain (14). Interestingly, the formation of PC is dependent on B-vitamin concentrations. In the homocysteine-methionine cycle, vitamin B6, vitamin B12, and folic acid are crucial in the conversion from homocysteine to methionine or cysteine. Insufficient concentrations of these $B$ vitamins result in the accumulation of homocysteine and its precursor, S-adenosyl homocysteine (SAH) (30). In turn, elevated SAH concentrations slow down phosphatidylethanolamine $\mathrm{N}$ methyltransferase, an enzyme responsible for the conversion of phosphatidylethanolamine to PC (12). This would imply that both B-vitamin and $\omega$-3 fatty acid concentrations should be high in cognitively impaired older subjects: B vitamins are needed to prevent accumulation of homocysteine and thereby stimulate the production of PC, and sufficient availability of $\omega-3$ fatty acids are needed because these are an important constituent of neuronal membranes $(30,31)$. As the amount of neuronal loss would be lower in cognitively healthy older adults, it could be that lower concentrations of $\omega-3$ fatty acids are sufficient to maintain synapses.

In addition to differences in $\omega-3$ fatty acid requirements, study populations might differ with respect to inflammatory states. We hypothesize that participants from the VITACOG and OmegAD trials had higher inflammatory states, as older age (32) and lower cognitive status (33) have been associated with higher inflammation concentrations. Inflammation is a key mechanism in the pathogenesis of age-related neurodegenerative diseases $(34,35)$. As both $\mathrm{B}$ vitamins and $\omega-3$ fatty acids exhibit anti-inflammatory properties (36), and nutrients may have complementary anti-inflammatory effects $(37,38)$, it could be argued that this group has higher requirements for antiinflammatory nutrients to counteract the harmful effects of elevated inflammation concentrations on cognition. To gain mechanistic insight into the role of inflammation in this interaction, future research could incorporate measurements of inflammation markers.

It is important to note that the opposing findings could also be attributed to differences in interventions or baseline nutrient statuses between study populations. Participants in our study were supplemented with folic acid only, while VITACOG participants received a combination of folic acid, vitamin B6, and vitamin B12. With respect to the baseline nutrient status, unfortunately, $\omega-3$ fatty acid concentrations cannot be compared directly between studies because of differences in the fatty acid fractions analyzed, analytical methods, and expressed measures. In our study population, the proportion of $\omega-3$ fatty acids $(\mathrm{EPA}+\mathrm{DHA})$ in plasma cholesteryl esters was $2.32 \pm 0.86 \%$. In the VITACOG trial, $\omega-3$ fatty acid concentrations (EPA + DHA) were expressed in absolute amounts and measured in plasma in free, phospholipid, triglyceride, and cholesteryl ester fractions, with an average of $472 \mu \mathrm{mol} / \mathrm{L}$ (95\% CI: 439, $508 \mu \mathrm{mol} / \mathrm{L})(14)$. In addition, homocysteine status, a possible factor in the mixed results of B-vitamin supplementation trials (39), was $\sim 2 \mu \mathrm{mol} / \mathrm{L}$ higher in our study compared to the previous trials. Vitamin B12 concentrations were comparable between study populations. This considerable role of the study population in the explanation of the opposite findings highlights the importance of a personalized approach in the field of nutrition and cognitive aging research.

We found that the effectiveness of folic acid treatment was dependent on the EPA tertile, but not on the DHA tertile. Our findings are in contrast to the VITACOG trial, in which DHA appeared to be driving the interaction between B-vitamin treatment and $\omega-3$ status (13). With regard to brain atrophy, the interaction was driven by both EPA and DHA (14). The above discussed differences between our trial and the VITACOG trial (study population, interventions, measures of fatty acids) could be responsible for the conflicting results.

A limitation of our study is that $\omega-3$ fatty acid concentrations were only assessed at baseline. However, previous research on the FACIT trial showed that there was a positive correlation between dietary intake of fish at baseline and after 3 y (6), suggesting that participants did not change their fish intakes. This limits influence of fish consumption on $\omega-3$ fatty acid plasma concentrations. It is also important to emphasize that the current analysis is a subgroup analysis; therefore, interpretation of results is limited. Strengths of our study include the long follow-up period of $3 \mathrm{y}$ and the use of an extensive battery of cognitive tests focusing on different domains.

Our analysis was limited to the interaction between folic acid and $\omega-3$ fatty acids. Yet, other nutrients may be involved in the interaction. In a post hoc analysis of the multidomain Alzheimer prevention trial (MAPT), Bowman and colleagues (40) developed a blood-based nutritional risk index and investigated its predictive quality on cognitive decline. Scores for the index ranged from 0 to 3 , with 1 point given for each suboptimal level of erythrocyte $\omega$-3 fatty acid, total plasma homocysteine, and/or serum 25-hydroxyvitamin D. Participants with optimal concentrations for all 3 factors showed cognitive improvements, while participants with suboptimal concentrations for $\geq 1$ of the factors declined over the study period, with each increase in the score leading to faster rates of cognitive decline. This suggests that B vitamins and $\omega-3$ fatty acids may not capture the full complexity of the interaction, and that vitamin $\mathrm{D}$ potentially plays a role as 
well. Unfortunately, vitamin D status was not measured in the current study. Future research should consider incorporating this measure, as well as an extensive blood nutrient assessment, to investigate the role of other dietary factors that have been linked to cognitive aging.

In conclusion, this post hoc analysis revealed that the effectiveness of folic acid treatment on cognitive functioning in healthy older adults is dependent on their $\omega$-3 fatty acid status. Healthy older adults with a lower $\omega-3$ status benefited from folic acid supplementation, while individuals with a higher $\omega-3$ fatty acid status did not show additional advantages of taking daily folic acid supplements. These results shed light on the presence of subgroups that benefit from B-vitamin supplementation, and emphasize the importance of a personalized approach. More research is needed to further disentangle the complex interaction between nutrition and cognitive aging, with a focus on investigating the roles of other nutrients and the underlying mechanisms.

The authors' responsibilities were as follows-OvdR, LCPGMdG: designed the research; OvdR: conducted the research; APMvS: analyzed the data and wrote the first draft of the manuscript; LCPGMdG: had primary responsibility for the final content; and all authors: contributed to the revision of the manuscript and read and approved the final manuscript.

The authors report no conflicts of interest.

\section{Data Availability}

Data described in the manuscript, code book, and analytic code will be made available upon request pending application and approval.

\section{References}

1. World Health Organization. Dementia key facts 2019. Geneva: World Health Organization; 2019. Available from: https://www.who.int/news -room/fact-sheets/detail/dementia.

2. Scarmeas N, Anastasiou CA, Yannakoulia M. Nutrition and prevention of cognitive impairment. Lancet Neurol 2018;17(11): $1006-15$.

3. Setién-Suero E, Suárez-Pinilla M, Suárez-Pinilla $\mathrm{P}$, Crespo-Facorro B, Ayesa-Arriola R. Homocysteine and cognition: A systematic review of 111 studies. Neurosci Biobehav Rev 2016;69:280-98.

4. Hu Q, Teng W, Li J, Hao F, Wang N. Homocysteine and Alzheimer's disease: Evidence for a causal link from Mendelian randomization. J Alzheimers Dis 2016;52(2):747-56.

5. Clarke R, Bennett D, Parish S, Lewington S, Skeaff M, Eussen SJ, Lewerin C, Stott DJ, Armitage J, Hankey GJ. Effects of homocysteine lowering with $\mathrm{B}$ vitamins on cognitive aging: Meta-analysis of 11 trials with cognitive data on 22,000 individuals. Am J Clin Nutr 2014;100(2):657-66.

6. Dullemeijer C, Durga J, Brouwer IA, Van De Rest O, Kok FJ, Brummer RJM, Van Boxtel MPJ, Verhoef P. n-3 Fatty acid proportions in plasma and cognitive performance in older adults. Am J Clin Nutr 2007;86(5):1479-85.

7. Schaefer EJ, Bongard V, Beiser AS, Lamon-Fava S, Robins SJ, $\mathrm{Au}$ R, Tucker KL, Kyle DJ, Wilson PWF, Wolf PA. Plasma phosphatidylcholine docosahexaenoic acid content and risk of dementia and Alzheimer disease: The Framingham Heart Study. Arch Neurol 2006;63(11):1545-50.

8. Rangel-Huerta OD, Gil A. Effect of omega-3 fatty acids on cognition: An updated systematic review of randomized clinical trials. Nutr Rev 2018;76(1):1-20.

9. Li MM, Yu JT, Wang HF, Jiang T, Wang J, Meng XF, Tan CC, Wang C, Tan L. Efficacy of vitamins B supplementation on mild cognitive impairment and Alzheimer's disease: A systematic review and metaanalysis. Curr Alzheimer Res 2014;11(9):844-52.
10. Ford AH, Almeida OP. Effect of vitamin B supplementation on cognitive function in the elderly: A systematic review and metaanalysis. Drugs Aging 2019;36(5):419-34.

11. Mazereeuw G, Lanctot KL, Chau SA, Swardfager W, Herrmann N. Effects of omega-3 fatty acids on cognitive performance: A metaanalysis. Neurobiol Aging 2012;33(7):1482.e17-29.

12. Selley ML. A metabolic link between S-adenosylhomocysteine and polyunsaturated fatty acid metabolism in Alzheimer's disease. Neurobiol Aging 2007;28(12):1834-9.

13. Oulhaj A, Jernerén F, Refsum H, Smith AD, de Jager CA. Omega-3 fatty acid status enhances the prevention of cognitive decline by $\mathrm{B}$ vitamins in mild cognitive impairment. J Alzheimers Dis 2016;50(2):547-57.

14. Jernerén F, Elshorbagy AK, Oulhaj A, Smith SM, Refsum H, Smith AD. Brain atrophy in cognitively impaired elderly: The importance of longchain $\omega-3$ fatty acids and B vitamin status in a randomized controlled trial. Am J Clin Nutr 2015;102(1):215-21.

15. Jernerén F, Cederholm T, Refsum H, Smith AD, Turner C, Palmblad J, Eriksdotter M, Hjorth E, Faxen-Irving G, Wahlund LO. Homocysteine status modifies the treatment effect of omega-3 fatty acids on cognition in a randomized clinical trial in mild to moderate Alzheimer's disease: The OmegAD Study. J Alzheimers Dis 2019;69(1):189-97.

16. Durga J, van Boxtel MP, Schouten EG, Kok FJ, Jolles J, Katan $\mathrm{MB}$, Verhoef P. Effect of 3-year folic acid supplementation on cognitive function in older adults in the FACIT trial: A randomised, double blind, controlled trial. Lancet North Am Ed 2007;369(9557): 208-16.

17. Jolles J, Houx P, Van Boxtel M, Ponds R. The Maastricht Aging Study: Determinants of cognitive aging. Maastricht, Netherlands: Neuropsych Publishers; 1995.

18. Van Der Elst W, Van Boxtel MPJ, Van Breukelen GJP, Jolles J. The concept shifting test: Adult normative data. Psychol Assess 2006;18(4):424-32.

19. Van Der Elst W, Van Boxtel MPJ, Van Breukelen GJP, Jolles J. The Stroop Color-Word test: Influence of age, sex, and education; And normative data for a large sample across the adult age range. Assessment 2006;13(1):62-79.

20. van der Elst W, van Boxtel MPJ, van Breukelen GJP, Jolles J. Rey's verbal learning test: Normative data for 1855 healthy participants aged 24-81 years and the influence of age, sex, education, and mode of presentation. J Int Neuropsychol Soc 2005;11(3):290-302.

21. Van Der Elst W, Van Boxtel M, Van Breukelen G, Jolles J. The letter digit substitution test: Normative data for 1,858 healthy participants aged 24-81 from the Maastricht Aging Study (MAAS): Influence of age, education, and sex. J Clin Exp Neuropsychol 2006;28(6): 998-1009.

22. Van Der Elst W, Van Boxtel MPJ, Van Breukelen GJP, Jolles J. Normative data for the animal, profession and letter $\mathrm{M}$ naming verbal fluency tests for Dutch speaking participants and the effects of age, education, and sex. J Int Neuropsychol Soc 2006;12(1):80-9.

23. Hixson JE, Vernier DT. Restriction isotyping of human apolipoprotein E by gene amplification and cleavage with HhaI. J Lipid Res 1990;31(3):545-8.

24. Ubbink JB, Hayward Vermaak WJ, Bissbort S. Rapid high-performance liquid chromatographic assay for total homocysteine levels in human serum. J Chromatogr B Biomed Sci Appl 1991;565(1-2): 441-6.

25. Ubbink JB, Serfontein WJ, De Villiers LS. Stability of pyridoxal-5phosphate semicarbazone: Applications in plasma vitamin B6 analysis and population surveys of vitamin B6 nutritional status. J Chromatogr B Biomed Sci Appl 1985;342(C):277-84.

26. R Core Team. R: A language and environment for statistical computing. Vienna, Austria: R Foundation for Statistical Computing; 2020.

27. Mi W, van Wijk N, Cansev M, Sijben JWC, Kamphuis PJGH. Nutritional approaches in the risk reduction and management of Alzheimer's disease. Nutrition 2013;29(9):1080-9.

28. Petralia RS, Mattson MP, Yao PJ. Communication breakdown: The impact of ageing on synapse structure. Ageing Res Rev 2014;14(1): $31-42$.

29. Scheff SW, Price DA, Schmitt FA, Mufson EJ. Hippocampal synaptic loss in early Alzheimer's disease and mild cognitive impairment. Neurobiol Aging 2006;27(10):1372-84.

30. Kumar A, Palfrey HA, Pathak R, Kadowitz PJ, Gettys TW, Murthy $\mathrm{SN}$. The metabolism and significance of homocysteine in nutrition and health. Nutr Metab 2017;14(1):78. 
31. Liu JJ, Green P, John Mann J, Rapoport SI, Sublette ME. Pathways of polyunsaturated fatty acid utilization: Implications for brain function in neuropsychiatric health and disease. Brain Res 2015;1597: 220-46.

32. Franceschi C, Capri M, Monti D, Giunta S, Olivieri F, Sevini F, Panourgia MP, Invidia L, Celani L, Scurti M. Inflammaging and anti-inflammaging: A systemic perspective on aging and longevity emerged from studies in humans. Mech Ageing Dev 2007;128(1): 92-105.

33. Bermejo P, Martín-Aragón S, Benedí J, Susín C, Felici E, Gil P, Ribera JM, Villar ÁM. Differences of peripheral inflammatory markers between mild cognitive impairment and Alzheimer's disease. Immunol Lett 2008;117(2):198-202.

34. Heneka MT, Carson MJ, El Khoury J, Landreth GE, Brosseron F, Feinstein DL, Jacobs AH, Wyss-Coray T, Vitorica J, Ransohoff RM. Neuroinflammation in Alzheimer's disease. Lancet Neurol 2015;14(4):388-405.

35. Kinney JW, Bemiller SM, Murtishaw AS, Leisgang AM, Salazar AM, Lamb BT. Inflammation as a central mechanism in Alzheimer's disease. Alzheimers Dement 2018;4:575-90.
36. Vasefi M, Hudson M, Ghaboolina-Zare E. Diet associated with inflammation and Alzheimer's disease. J Alzheimers Dis Rep 2019;3(1):299-309.

37. Frith E, Shivappa N, Mann JR, Hébert JR, Wirth MD, Loprinzi PD. Dietary inflammatory index and memory function: Population-based national sample of elderly Americans. Br J Nutr 2018;119(5):552-8.

38. Kesse-Guyot E, Assmann KE, Andreeva VA, Touvier M, Neufcourt L, Shivappa N, Hébert JR, Wirth MD, Hercberg S, Galan P. Longterm association between the dietary inflammatory index and cognitive functioning: Findings from the SU. VI. MAX study. Eur J Nutr 2017;56(4):1647-55.

39. de Jager CA, Oulhaj A, Jacoby R, Refsum H, Smith AD. Cognitive and clinical outcomes of homocysteine-lowering B-vitamin treatment in mild cognitive impairment: A randomized controlled trial. Int J Geriatr Psychiatry 2012;27(6):592-600.

40. Bowman GL, Dodge HH, Guyonnet S, Zhou N, Donohue J, Bichse A, Schmitt J, Hooper C, Bartfai T, Andrieu S. A blood-based nutritional risk index explains cognitive enhancement and decline in the multidomain Alzheimer prevention trial. Alzheimers Dement 2019;5:953-63. 